\title{
A high sensitivity momentum flux measuring instrument for plasma thruster exhausts and diffusive plasmas
}

\author{
Michael D. West, ${ }^{\text {a) }}$ Christine Charles, and Rod W. Boswell \\ Space Plasma, Power and Propulsion Group, Research School of Physics and Engineering, \\ The Australian National University, Canberra ACT 0200, Australia
}

(Received 26 January 2009; accepted 4 May 2009; published online 28 May 2009)

\begin{abstract}
A high sensitivity momentum flux measuring instrument based on a compound pendulum has been developed for use with electric propulsion devices and radio frequency driven plasmas. A laser displacement system, which builds upon techniques used by the materials science community for surface stress measurements, is used to measure with high sensitivity the displacement of a target plate placed in a plasma thruster exhaust. The instrument has been installed inside a vacuum chamber and calibrated via two different methods and is able to measure forces in the range of $0.02-0.5 \mathrm{mN}$ with a resolution of $15 \mu \mathrm{N}$. Measurements have been made of the force produced from the cold gas flow and with a discharge ignited using argon propellant. The plasma is generated using a Helicon Double Layer Thruster prototype. The instrument target is placed about 1 mean free path for ion-neutral charge exchange collisions downstream of the thruster exit. At this position, the plasma consists of a low density ion beam (10\%) and a much larger downstream component $(90 \%)$. The results are in good agreement with those determined from the plasma parameters measured with diagnostic probes. Measurements at various flow rates show that variations in ion beam velocity and plasma density and the resulting momentum flux can be measured with this instrument. The instrument target is a simple, low cost device, and since the laser displacement system used is located outside the vacuum chamber, the measurement technique is free from radio frequency interference and thermal effects. It could be used to measure the thrust in the exhaust of other electric propulsion devices and the momentum flux of ion beams formed by expanding plasmas or fusion experiments. (C) 2009 American Institute of Physics. [DOI: 10.1063/1.3142477]
\end{abstract}

\section{INTRODUCTION}

Various instruments have been developed previously to measure the momentum flux of plasma flows, diffusive plasmas, and ion beams. A capacitance manometer has been used for momentum measurements in atomic and charged particle jets. ${ }^{1}$ It is a complex device consisting of a refractory target plate attached by a long shaft to a thin, tensioned, circular, metallic diaphragm that is one electrode of a capacitor from a baratron pressure sensor. The shaft is suspended by two wires and the whole system behaves like a pendulum with the pressure transducer acting as a displacement sensor. A compact cantilever force probe based on the pull-in phenomenon in microelectromechanical-system electrostatic actuators has been recently employed for plasma pressure measurements in the ISTTOK tokamak. ${ }^{2}$ Ballistic pendulums have been employed for momentum measurements of laserproduced plasmas, which are of interest to the fusion research community, for many years. ${ }^{3,4}$ These pendulums consist of a planar target, often made of Mylar or brass, suspended by nylon threads. Various techniques, including shadowgraphy and Hall effect sensors, have been used to determine the displacement of the pendulum and hence the momentum of the plasma flow. None of these systems adequately deal with the problems of electromagnetic interfer-

\footnotetext{
${ }^{\text {a)} E l e c t r o n i c ~ m a i l: ~ m i c h a e l . w e s t @ a n u . e d u . a u . ~}$
}

ence and large temperature changes commonly produced by magnetoplasmadynamic thrusters or radio frequency driven plasmas.

A double pendulum system, consisting of two identical plates suspended on razor sharp edges, has been used to determine the momentum flux and hence the thrust at the exhaust of a tandem mirror plasma thruster. ${ }^{5}$ The change in capacitance between the two plates was measured as a voltage signal, whose amplitude was proportional to the impacting force or impulse from the plasma thruster. Thrust levels of approximately $75 \mathrm{mN}$ were measured in this case. More recently, a target plate placed in the VASIMR plasma thruster's exhaust has been used to measure thrust levels of the order of tens of millinewtons. ${ }^{6,7}$ Attached to the target plate is an alumina rod, which is then fixed to a titanium beam on which four strain gauges are mounted. The force from the thruster's exhaust on the target plate generates a torque, causing the strain in the beam that is detected by the gauges in a Wheatstone bridge configuration. A sensitivity of $0.1 \mathrm{mN}$ up to a force of $270 \mathrm{mN}$ is claimed. ${ }^{8}$ This technique uses semiconductor strain gauges that are custom manufactured, particularly difficult to mount, and also must be shielded from temperature changes and electromagnetic interference. An instrument based on that same design has also been adapted for plasma pressure measurements in the ISTTOK tokamak. ${ }^{9}$

Expanding plasmas and ion beams are often used for the 
processing of materials and the growth of thin films on substrates. ${ }^{10}$ Several techniques have been developed to measure the stress induced curvature or bending of thin film substrate combinations during plasma processing. ${ }^{11}$ The deformation of the substrate resulting from tensile or compressive stress in the plasma deposited amorphous thin film can be detected electrically or optically, but optical methods are preferred as they are not sensitive to the electric and magnetic fields or the charge up of the substrate. Systems developed to date include those that use beam splitters ${ }^{12}$ and position sensitive detectors. ${ }^{13}$ Recently, a simplified technique has been developed, which dispenses with some of the mirrors and electronics required by the above systems and has been used to provide continuous stress measurements during film growth produced by a helicon activated reactive evaporation deposition system. ${ }^{14}$

Laser interferometry and other systems using laser measurements have also been employed to measure the displacement and dynamics of thrust balances. By placing the measurement system outside the vacuum chamber, the need for vacuum compatible optical components is removed and the problems associated with electromagnetic interference and thermal fluctuations are minimized. This approach has been used for the measurement of thrust and impulse bit of several high-power steady-state plasma thrusters ${ }^{15-17}$ and micropropulsion thrusters. ${ }^{15,18-20}$ High accuracy ( $<2 \%$ error) thrust measurements within a very wide range of impulses (100 $\mu$ Ns to above $10 \mathrm{Ns}$ ) have been reported. ${ }^{15}$

In this work, we report on the development of a momentum flux measuring instrument (MFMI) based on a compound pendulum that builds upon the techniques used by the materials science community for surface stress measurements. By adapting a simplified optical deflection technique, ${ }^{14}$ the displacement of a target plate placed in a plasma thruster exhaust or diffusive plasma can be measured with a high sensitivity. This enables higher resolution momentum flux measurements of plasmas produced by electric propulsion devices and $\mathrm{rf}$ driven plasmas. This new technique uses a simple and inexpensive target plate and places the measurement technique outside the vacuum chamber. Therefore, the laser displacement system does not need shielding from thermal effects, a vacuum compatible displacement sensor is not required, and problems caused by vibration and electromagnetic interference are minimized. Since only one window is required to point the laser at the instrument target plate, this technique can be easily adapted to other apparatuses if required. This approach does, however, require the target plate to be made from a vacuum compatible material.

\section{INSTRUMENT SETUP}

\section{A. Construction}

The MFMI, as shown in Fig. 1, is a compound pendulum that consists of a target plate, in this case a 6 in. silicon wafer (150 $\mathrm{mm}$ in diameter), attached to an alumina rod-so it is electrically isolated-and then to a stainless steel tube, $3.75 \mathrm{~mm}$ in diameter. The alumina rod slides inside the stainless steel tubing and is held in place with a small grub screw.

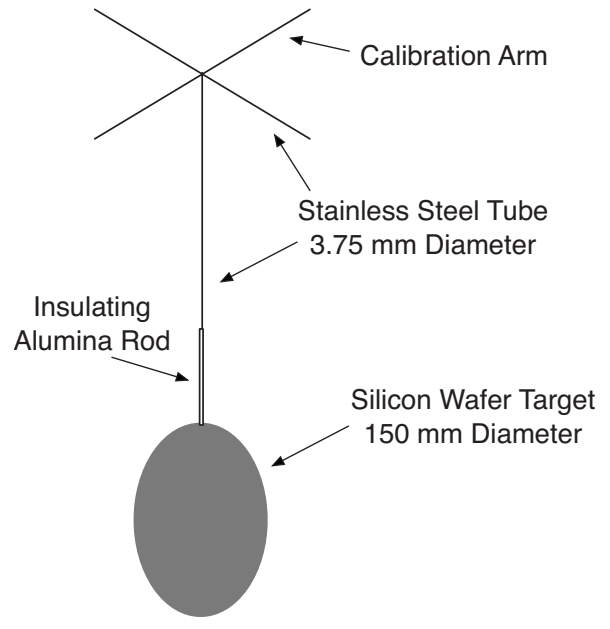

FIG. 1. Construction of the MFMI.

This allows the length of the instrument to be adjusted as required and target plates to be interchanged easily if required. Attached perpendicular to the tubing is a crosspiece also made from stainless steel tube $3.75 \mathrm{~mm}$ in diameter which pivots on two machined knife edges affixed to the roof of the test facility described later. Another piece of stainless steel tube $3.5 \mathrm{~mm}$ in diameter is attached perpendicular to the crosspiece and is $200 \mathrm{~mm}$ long. This piece provides a $100 \mathrm{~mm}$ moment arm on each side of the pivot point for calibration purposes. One side of the silicon wafer, which is used as the target plate, has a polished surface that can be used to reflect the laser beam. This allows a nonintrusive measurement of the displacement of the instrument. The total mass of the instrument, that is, the target, alumina rod, and stainless steel tubing, was found to be $67.948 \pm 0.001 \mathrm{~g}$.

\section{B. Test facility and laser measurement system}

The test facility used is a stainless steel vacuum chamber $1 \mathrm{~m}$ in diameter and $1.4 \mathrm{~m}$ long that houses the Helicon Double Layer Thruster (HDLT) prototype, as described recently. ${ }^{21}$ A turbomolecular/rotary pumping system maintains a base pressure of less than $9 \times 10^{-7}$ Torr with an effective pumping speed measured for argon of approximately $3301 \mathrm{~s}^{-1}$. The HDLT prototype, which is described in detail elsewhere, ${ }^{21,22}$ is installed inside the vacuum chamber, as shown in Fig. 2. The end of the HDLT structure is at $z=0 \mathrm{~cm}$, and the end of the HDLT source tube and hence the exit of the thruster are at $z=3 \mathrm{~cm}$. In this study, the HDLT prototype is simply used as a plasma source to provide the flowing plasma and ion beam used to test the MFMI. The rf power is limited to $100 \mathrm{~W}$ in this work to provide low density plasma and low momentum flux on the target since the main objective is to test the sensitivity and maximum resolution of the developed instrument. The plasma produced by HDLT prototype has been extensively characterized previously. $^{21}$

The MFMI is placed on the two machined knife edges that are suspended from rails attached to the roof on the inside of the vacuum chamber. Vibration isolation between the knife edges and the vacuum chamber was considered; however, the vibration caused by the pumping system was 


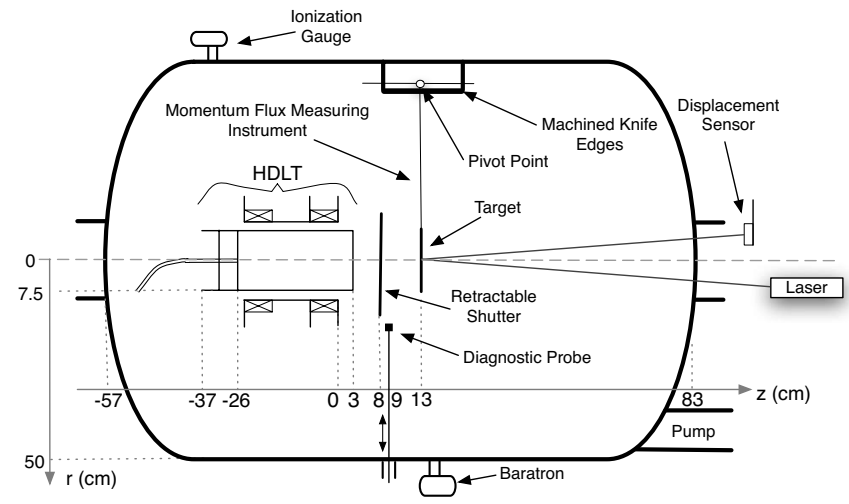

FIG. 2. Schematic of the vacuum chamber from the side with the HDLT prototype, MFMI, and laser measurement system installed. A diagnostic probe is also installed via a side port at $z=9 \mathrm{~cm}$.

found to be less than anticipated and negligible. The MFMI is positioned so that the target is $100 \mathrm{~mm}$ from the end of the HDLT source tube at $z=13 \mathrm{~cm}$, as shown in Fig. 2. Using a method similar to that employed previously, ${ }^{14}$ the displacement of the target plate is measured using a $1 \mathrm{~mW} \mathrm{He}-\mathrm{Ne}$ laser $(\lambda=633 \mathrm{~nm})$ pointed at the back of the silicon wafer target. The displacement of the reflected beam spot is measured using a THORLABS PDQ80S1 position sensing system that utilizes a quadrant photodetector operating in the visible to near-IR range. A series of lenses focuses the laser beam, which then passes through a large window port at the downstream end of the vacuum chamber, reflects off the MFMI target, and passes back through the window port and onto the position sensor. A USM (Universal Serial Bus) interface sends the $x$ and $y$ coordinates of the center of the reflected beam spot from the photodetector to a computer for data analysis.

To minimize the oscillations caused by the surge of propellant striking the MFMI target when the propellant is injected prior to igniting the HDLT, a Millipore Tylan WGFG inline filter is installed on the propellant line between the mass flow controller and the nylon tubing that feeds the propellant into the vacuum chamber and then into the HDLT source tube. In addition, a shutter, made from a disk of stainless steel sheet $1 \mathrm{~mm}$ thick and $160 \mathrm{~mm}$ in diameter, is placed between the end of the HDLT source tube and the MFMI target plate. The shutter is located $50 \mathrm{~mm}$ from the end of the HDLT source at $z=8 \mathrm{~cm}$ and is attached to a stainless steel rod that enables the shutter to be retracted radially and removed from the HDLT exhaust plume. This rod includes a ceramic break that electrically isolates the shutter disk and ensures that it is floating. This allows for the propellant flow to be switched on, the HDLT to be ignited, and the plasma formed without causing the MFMI to oscillate. Without these modifications the instrument would undergo large oscillations that take several hours to damp out since it moves in an evacuated volume and the only source of damping is the friction where the MFMI sits atop the machined knife edges.

\section{Plasma diagnostics}

A retarding field energy analyzer (RFEA) is installed radially $60 \mathrm{~mm}$ from the exit of the HDLT source tube at

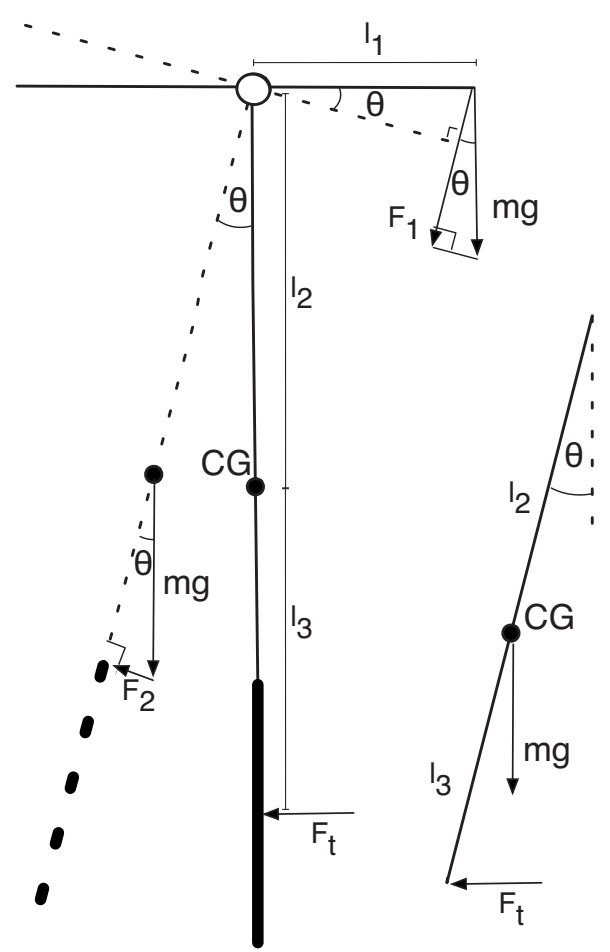

FIG. 3. Free body diagram for MFMI calibration. Main: forces applied in relation to the pivot point of the MFMI. Inset: forces applied in relation to the CG of the MFMI.

$z=9 \mathrm{~cm}$. This RFEA can be moved radially from the HDLT centerline $(r=0 \mathrm{~cm})$ to the wall of the vacuum chamber $(r=50 \mathrm{~cm})$ and can also be rotated upon its support tube axis so measurements can be made with the entrance orifice facing the HDLT exhaust $\left(\theta=0^{\circ}\right)$ or the chamber walls $\left(\theta=90^{\circ}\right)$. The RFEA is based on earlier designs ${ }^{23,24}$ and is used to determine the ion energy distribution function and characterize the plasma and ion beam created by the HDLT. Full details of the integration of the RFEA with this test facility and the data collection and analysis techniques are available elsewhere. ${ }^{21}$ Measurements are made with the radial RFEA inserted between the MFMI and the end of the HDLT source at $z=9 \mathrm{~cm}$. A planar disk Langmuir probe $3 \mathrm{~mm}$ in diameter is also installed radially to measure the plasma density in front of and behind the MFMI target. The Langmuir probe can be moved radially from $r=0 \mathrm{~cm}$ to $r$ $=20 \mathrm{~cm}$ and can be positioned in front of (at $z=12 \mathrm{~cm}$ ) or behind (at $z=14 \mathrm{~cm}$ ) the MFMI target as desired.

\section{CALIBRATION}

To calibrate the MFMI, known masses were added to the calibration arm of the MFMI while the test facility was open and at atmospheric pressure, and the displacement of the target was measured using the laser displacement sensor. The displacement of the MFMI instrument was measured for several different masses ranging from 8.567 to $177.267 \mathrm{mg}$. The free body diagrams shown in Fig. 3 are used to derive how the mass applied to the moment arm $m$ can be related to the force on the target plate $F_{t}$. Since the moments around the pivot point $(\bigcirc)$ equal zero,

$$
F_{1} l_{1}-F_{2} l_{2}=0 \text {, }
$$




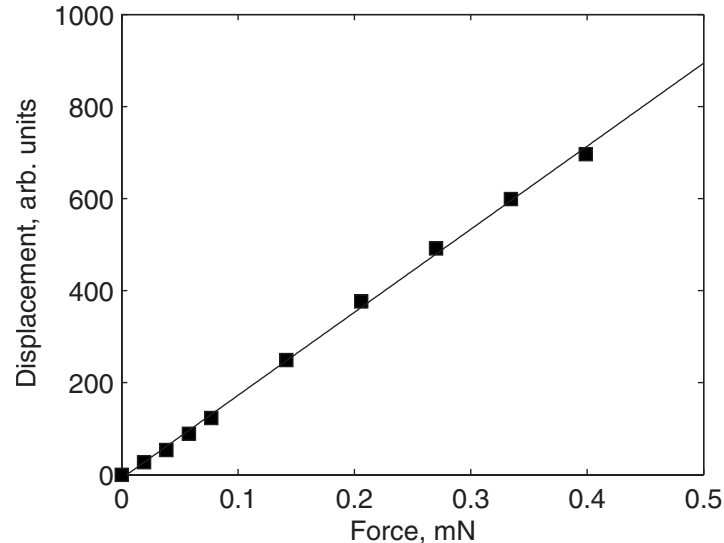

FIG. 4. Typical calibration curve for the MFMI using known masses applied to the calibration arm.

$$
\begin{aligned}
& m g \cos \theta l_{1}=M g \sin \theta l_{2}, \\
& \therefore \tan \theta=\frac{m g l_{1}}{M g l_{2}}, \\
& \therefore \theta=\tan ^{-1}\left[\frac{m g l_{1}}{M g l_{2}}\right] .
\end{aligned}
$$

The moments around the center of gravity (O) shown in the inset of Fig. 3 also equal to zero such that

$$
\begin{aligned}
& -M g \sin \theta l_{2}+F_{t}\left(l_{2}+l_{3}\right)=0, \\
& \therefore F_{t}=M g \sin \theta \frac{l_{2}}{l_{2}+l_{3}} .
\end{aligned}
$$

Combining Eqs. (4) and (6) yields

$$
F_{t}=M g \sin \left\{\tan ^{-1}\left[\frac{m g l_{1}}{M g l_{2}}\right]\right\} \frac{l_{2}}{l_{2}+l_{3}},
$$

where $g$ is the acceleration due to gravity, $l_{1}$ is the length from the pivot point to the moment arm $\left(l_{1}=100 \mathrm{~mm}\right), l_{2}$ is the length from the pivot point to the center of gravity of the instrument $\left(l_{2}=210 \mathrm{~mm}\right)$, and $l_{3}$ is the length from the center of gravity to the center of the target plate $\left(l_{3}=226 \mathrm{~mm}\right)$.

Figure 4 shows a typical calibration curve for the MFMI and reveals a linear relationship between the force applied to the target $F_{t}$ and the displacement of the laser beam spot $\Delta y$ measured by the laser displacement sensor. Since the $y$-axis position determined by the quadrant photodetector is computed by taking the difference in the voltage measured by the top and bottom quadrants and is then expressed as a digital value between \pm 1500 by the software of the positioning system, $\Delta y$ is expressed in arbitrary units. In the current configuration, the MFMI is capable of measuring forces on the target of up to $0.5 \mathrm{mN}$ with a resolution of $15 \mu \mathrm{N}$. The resolution of the MFMI is limited by the resolution of the photodetector, the size of the beam spot that is reflected off the polished side of the target, and the mass of the MFMI. Vibrations caused by the vacuum pumps and other equipments associated with the experiment, such as power supplies and cooling fans, could also limit the resolution of the MFMI yet were not found to be a concern in this case. The maximum force measured by the MFMI is limited by how much the
MFMI can oscillate before it strikes the thruster and the range of displacements that can be measured by the laser measurement system. The latter can easily be increased if necessary by decreasing the size of the reflected beam spot using the focusing lenses or by using a photodetector with a larger sensor area.

\section{METHODOLOGY AND DATA ANALYSIS}

The reflected laser beam spot from the back of the MFMI target is aligned so that it is at the center of the laser displacement sensor when the MFMI is at rest. Prior to recording data with the laser displacement sensor the equipment required to operate the HDLT is turned on, including the solenoid power supply, the rf generator, and the standing wave reflected and power meter and oscilloscope that are used for monitoring the inputted rf power. These are switched on to ensure that any small vibrations caused by the operation of these devices are present throughout the measurement process at a known point in time. With the shutter inserted to cover the open end of the HDLT source tube, the recording of data then commences. After $90 \mathrm{~s}$, the propellant is injected and the rf power switched on to ignite the plasma. In the case where the response from only the propellant flow is measured, the procedure is identical except that the $\mathrm{rf}$ power is not switched on and no plasma is formed. The tuning on the HDLT matching box is adjusted to ensure that the plasma is coupled correctly and sufficient rf power is deposited in the plasma. At $150 \mathrm{~s}$, the shutter is retracted, which allows the plasma to strike the MFMI. The shutter remains retracted for $120 \mathrm{~s}$, allowing the MFMI to oscillate and reach a new equilibrium position in response to the force applied to it by the plasma (or the propellant flow). At $270 \mathrm{~s}$, the shutter is inserted again and the plasma is left on for a further $60 \mathrm{~s}$, at which point the rf power and propellant flow are switched off. The laser displacement sensor continues to record data for a further $150 \mathrm{~s}$. The total recording time is $480 \mathrm{~s}$. The MFMI, which now oscillates as a result of the force from the plasma (or propellant flow), then requires approximately $3 \mathrm{~h}$ to return to rest due to the limited friction in the system. Several devices were considered to dampen the oscillations and reduce the time between measurements but all were likely to result in movement of the MFMI from its intended position and affect the alignment of the reflected laser beam spot on the photodetector and the accuracy of the subsequent measurements.

Figure 5 shows an example of the raw $y$-axis displacement data collected by the laser displacement sensor versus time. Since the oscillation frequency of the MFMI is high, the data appear very noisy yet the displacement of the MFMI is clear when the shutter is retracted at $150 \mathrm{~s}$. To determine the displacement of the MFMI caused by the conditions investigated, the data are smoothed using a ten point moving average. This filter window amounts to a $10 \mathrm{~s}$ period. This is greater than the period of oscillation of the MFMI, which is about $4 \mathrm{~s}$. Figure 5 also shows the data obtained following smoothing. This method has been applied to all data sets presented here. To determine the displacement resulting from the conditions tested, $\Delta y$ is the average displacement be- 


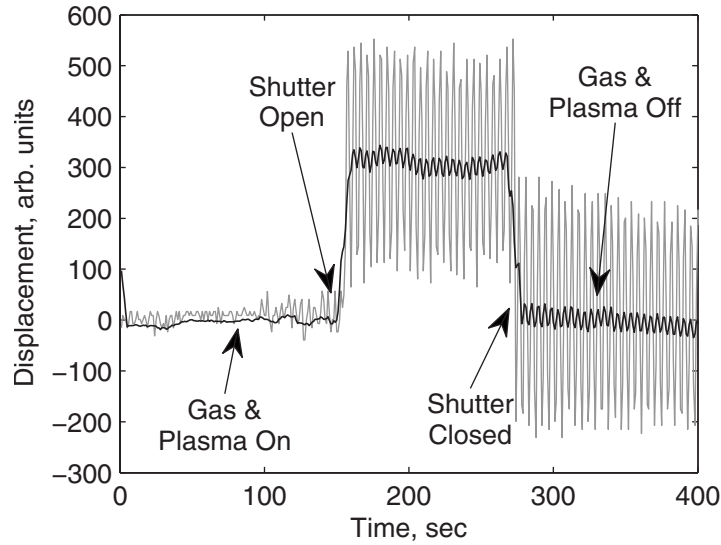

FIG. 5. The raw data produced from the laser displacement sensor (grey line) and the raw data following smoothing (black line). The labels highlight when the propellant and plasma are turned on and off and when the shutter is opened and closed.

tween $t=160 \mathrm{~s}$ and $t=260 \mathrm{~s}$, that is, $10 \mathrm{~s}$ after the shutter is retracted, which allows for the MFMI to recover from the effect of retracting the shutter, to $10 \mathrm{~s}$ before it is closed. For the example in Fig. 5, the mean is 309.1 units with a standard deviation of 10.7 units. Therefore, $\Delta y=309.1 \pm 10.7$ units. Using the calibration curve (Fig. 4) the displacement measured is converted into the force measured on the target $F_{t}$. For the case shown in Fig. 5, where $\Delta y=309.1 \pm 10.7$ units, $F_{t}=0.176 \pm 0.006 \mathrm{mN}$.

\section{EXPERIMENTAL RESULTS AND DISCUSSION}

\section{A. Cold gas (no discharge)}

Before investigating the force measured by the MFMI with the HDLT operating, it is important to understand the forces acting on the target of the MFMI from the background pressure in the vacuum chamber and from the flow of neutral propellant exiting the thruster. Three cases are considered: (1) the forces applied by the background pressure with no directed flow, (2) the forces applied when an underexpanded sonic orifice is used to inject a propellant flow directly at the MFMI target, and (3) the forces measured when the propellant flow is injected at the end of the HDLT source tube at a distance from the MFMI target. When no propellant flow is directed at the MFMI target yet the background pressure is increased, the background pressure exerts an equal force on the front and back sides of the MFMI target, $F_{\mathrm{bg}}^{+}$and $F_{\mathrm{bg}}^{-}$, respectively, as shown in Fig. 6(a). Here $F_{t}=F_{\mathrm{bg}}^{+}+F_{\mathrm{bg}}^{-}=0$.

To investigate the second case, measurements were made using the MFMI of the force generated by the cold gas flow through an underexpanded orifice placed close to the MFMI target, as shown in Fig. 6(b). These measurements also help to confirm the accuracy of the calibration outlined above. A stainless steel tube, with one end attached to the mass flow controller that controls the propellant flow and the other attached to a machined orifice $3 \mathrm{~mm}$ in diameter pointing perpendicular to the centerline of the tube, was inserted radially through another side port on the vacuum chamber. It was positioned such that the orifice was $1 \mathrm{~cm}$ from the MFMI target at $z=12 \mathrm{~cm}$ and at $r=0 \mathrm{~cm}$ (i.e., on the HDLT and vacuum chamber centerline). With the orifice facing
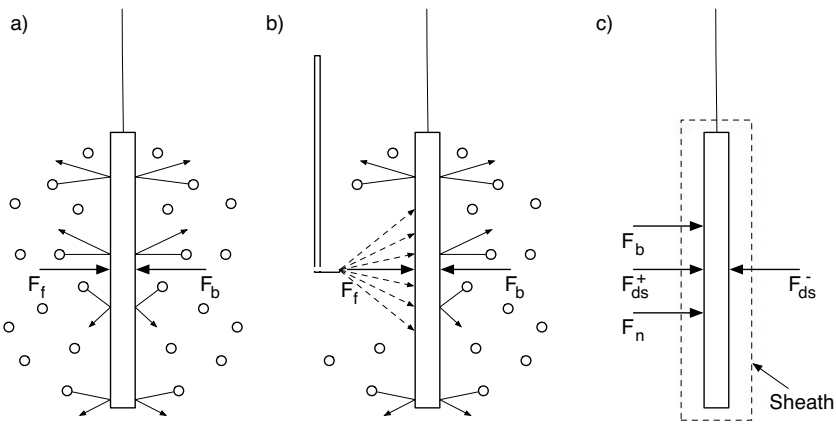

FIG. 6. Forces acting on the MFMI target (a) from the background gas, (b) from the background gas and the jet from the underexpanded orifice, and (c) from the ion beam, the downstream plasma and the background gas when the plasma discharge is ignited. The sheath thickness in (c) is less than $1.5 \mathrm{~mm}$.

away from the HDLT source and pointed at the MFMI target, measurements were made at various flow rates using argon.

In this case, the force on the target is $F_{t}=F_{j}+F_{\mathrm{bg}}^{+}+F_{\mathrm{bg}}^{-}$, where $F_{j}$ is the force from the jet flow from the orifice. Since, $F=\bar{v}_{n}(\Delta m / \Delta t)$, the force from the jet flow is given by

$$
F_{j}=\alpha_{j} n_{n} A_{t} m_{n} \bar{v}_{n}^{2},
$$

where $\alpha_{j}$ is an empirical experimental gas transmission coefficient for the jet flow that is determined by a line of best fit, $n_{n}$ is the neutral gas density, $A_{t}$ is the total area of the MFMI target, and $m_{n}$ is the neutral gas particle mass. The average velocity of the neutral gas particles $\bar{v}_{n}$ is found from $\bar{v}_{n}=\sqrt{8 R T / \pi M}$, where $R$ is the ideal gas constant, $T$ is the gas temperature, and $M$ is the molecular weight of the gas. The neutral gas density is found from the ideal gas law, $n_{n}=p V / k T$. Since $F_{\mathrm{bg}}^{+}$and $F_{\mathrm{bg}}^{-}$are equal, the force on the MFMI $F_{t}$ equals $F_{j}$. Figure 7 shows the results of measurements made for this case at varying flow rates. The force measured on the target is shown as filled circles and the solid line is the calculated value of $F_{j}$ when $\alpha_{j}=0.295$.

With the orifice removed, measurements were then made with the propellant flow injected at the end of the HDLT source tube. As mentioned previously and shown in Fig. 2, the propellant line enters the closed end of the HDLT source

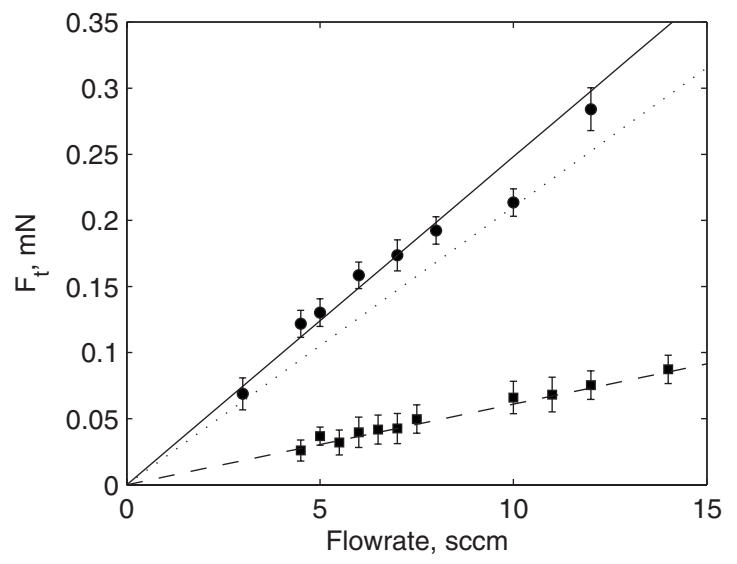

FIG. 7. Force on the target from propellant flow using an underexpanded orifice (filled circle) and with propellant injected at the closed end of the HDLT source tube (filled square). The solid line is the calculated value of $F_{j}$ when $\alpha_{j}=0.295$, the dashed line is the calculated value when $\alpha_{j}=0.0725$ and the dotted line is the calculated value when $\alpha_{j}=0.25$. 
at $z=-26 \mathrm{~cm}$. The propellant line is made from a nylon tubing with an internal diameter of $4.2 \mathrm{~mm}$. The propellant is injected $29 \mathrm{~cm}$ from the exit of the HDLT source and $39 \mathrm{~cm}$ from the MFMI target. The force on the target in this case is $F_{t}=F_{n}+F_{\text {bg }}^{+}+F_{\text {bg }}^{-}$, where $F_{n}$ is the force from the neutral propellant flow injected into the HDLT source tube. Again, $F_{\mathrm{bg}}^{+}$ and $F_{\text {bg }}^{-}$are equal and

$$
F_{n}=\alpha_{n} n_{n} A_{t} m_{n} \bar{v}_{n}^{2},
$$

where $\alpha_{n}$ is an empirical experimental gas transmission coefficient for the injected propellant. The filled squares in Fig. 7 show the force measured by the MFMI for different propellant flow rates for this case. The calculated value of $F_{n}$ is shown as the dashed line in Fig. 7 when $\alpha_{n}=0.0725$.

As expected the force measured increases with increasing flow rate; however, the magnitude of the force is significantly lower than that measured earlier using the underexpanded orifice placed at $1 \mathrm{~cm}$ from the MFMI target, as described above. This difference can be accounted for by the decrease in flux and velocity caused by the collisions that the neutral particles experience while traveling the $39 \mathrm{~cm}$ from the exit of the nylon tubing to the MFMI target and the diffusion of the neutral particles into the vacuum chamber once they exit the HDLT source tube. This decrease can be quantified by the ratio of the gas transmission coefficients for the two cases, $\alpha_{j} / \alpha_{n} \approx 4$. Therefore, the propellant injected through the underexpanded orifice positioned $1 \mathrm{~cm}$ from the MFMI produces four times the force produced when the propellant is injected at the closed end of the HDLT source tube $39 \mathrm{~cm}$ from the MFMI. In summary, the force from the cold gas flow has been measured when the gas is injected close to the MFMI and from the upstream end of the source tube. The force produced by the cold gas has been shown to increase with flow rates in a repeatable fashion for both cases, as expected.

\section{B. Hot plasma (discharge on)}

When the HDLT is switched on and a plasma formed, the force on the MFMI target $F_{t}$ is the sum of the force from the neutral propellant flow $F_{n}$ and the force from the ions and electrons of the plasma impacting the target $F_{p}$. Since the electrons are much lighter than the ions, the force from the electrons is considered negligible. The ions impacting the front side of the MFMI target consist of a population of slow ions and a population of fast ions from the ion beam. The back of the MFMI target is impacted by the slow ion population only. ${ }^{25}$ Ions in the local downstream plasma are accelerated by sheaths that form at both the front and back sides of the MFMI target. The width of the sheath is less than $1.5 \mathrm{~mm}$. These local ions enter the sheath at the Bohm velocity $v_{\text {Bohm }}$ and the presheath acceleration potential is about $T_{e} / 2$, which is much smaller than the sheath potential $V_{\text {sheath }}$, which is approximately $35 \mathrm{~V}$. The presheath acceleration is directed normal to the sheath and therefore we assume that the pitch angle of the local plasma ions impacting the target plate is zero, i.e., all the ions impact the target perpendicular to the surface of the target. The resulting opposite forces acting on the MFMI target can be written as

$$
\begin{aligned}
& F_{\mathrm{ds}}^{+}=n_{\mathrm{ds}}^{+} m_{i} A_{p} v_{\mathrm{Bohm}}^{2}, \\
& F_{\mathrm{ds}}^{-}=n_{\mathrm{ds}}^{-} m_{i} A_{p} v_{\mathrm{Bohm}}^{2},
\end{aligned}
$$

where $n_{\mathrm{ds}}^{+}$and $n_{\mathrm{ds}}^{-}$are the density of the downstream plasma in front of and behind the target plate, respectively, and $m_{i}$ is the mass of an argon ion. $A_{p}$ is the area of the plasma that impacts the target plate. Radial measurements with the Langmuir probe in front of and behind the target plate show that for the conditions investigated here, the plasma density is constant from $r=0 \mathrm{~cm}$ to $r=6.5 \mathrm{~cm}$ and drops off sharply at $r>6.5 \mathrm{~cm}$. The percentage change in the plasma density over this radius, from $r=0 \mathrm{~cm}$ to $r=6.5 \mathrm{~cm}$, is less than $5 \%$. $A_{p}$ is therefore calculated assuming a radius of $6.5 \mathrm{~cm}$.

In the present case where an ion beam is present, ${ }^{21}$ these ions also impact the target plate. The force produced by the beam ions is given by

$$
F_{b}=n_{b} m_{i} A_{p} v_{b}^{2}
$$

where $n_{b}$ is the ion beam density and $v_{b}$ is the velocity of the beam ions which can be determined by

$$
v_{b}=\sqrt{\frac{2 e V_{b}}{m_{i}}},
$$

where $e$ is the electron charge and $V_{b}$ is the ion beam potential. The sheath in front of the MFMI is probably dominated by the large population of ions from the local plasma (90\%). The additional ion beam population $(10 \%)$ originate from a region well upstream inside the HDLT source where the plasma potential is near $V_{b}{ }^{26,27}$ These ions suffer no collisions and acquire the full potential drop of $50 \mathrm{~V}$ between that region and the target. Since it is an expanding plasma, it is possible that these ions "enter" the region of $V_{p}=50 \mathrm{~V}$ with a Bohm velocity. However, this would correspond to a potential drop of about $T_{e} / 2$, that is, only a few volts and is neglected in the calculation of $v_{b}$ from Eq. (13). In a helicon source similar to the one used here, it has been shown that approximately $98 \%$ of the momentum of the beam ions produced is parallel to the centerline of the source. ${ }^{28}$ Therefore it is assumed that the beam ions here have a zero pitch angle, that is, the beam ions impact the target perpendicular to the surface of the target plate. The net force on the plate from the plasma, which can be determined from the plasma parameters just outside the sheath regions, is

$$
F_{p}=n_{\mathrm{ds}}^{+} m_{i} A_{p} v_{\mathrm{Bohm}}^{2}-n_{\mathrm{ds}}^{-} m_{i} A_{p} v_{\mathrm{Bohm}}^{2}+n_{b} m_{i} A_{p} v_{b}^{2},
$$

and therefore, as shown in Fig. 6(c), the total force exerted on the target when the HDLT is switched on and a plasma formed is

$$
\begin{aligned}
F_{t}= & n_{\mathrm{ds}}^{+} m_{i} A_{p} v_{\mathrm{Bohm}}^{2}-n_{\mathrm{ds}}^{-} m_{i} A_{p} v_{\mathrm{Bohm}}^{2}+n_{b} m_{i} A_{p} v_{b}^{2} \\
& +\alpha_{n} n_{n} A_{t} m_{n} \bar{v}_{n}^{2} .
\end{aligned}
$$

The ions in the beam impact the MFMI target at a velocity dictated by the beam potential. Using the radial RFEA positioned in front of the MFMI target plate with the orifice facing the HDLT exhaust $\left(\theta=0^{\circ}\right)$, the ion energy distribution function is measured. This is used to determine the ion beam and local downstream plasma potentials $V_{b}$ and $V_{\mathrm{ds}}$, respectively, and the ratio of the ion beam density to the down- 
stream plasma density $n_{b} / n_{\mathrm{ds}}{ }^{29}$ When operating at $100 \mathrm{~W}$ of rf power at $13.56 \mathrm{MHz}$, with a propellant flow rate of $10 \mathrm{sccm}$ (SCCM denotes cubic centimeters per minute at standard temperature and pressure) of argon, resulting in a pressure of 0.38 mTorr and with a maximum axial dc magnetic field $B_{z}$ of $138 \mathrm{G}$ inside the HDLT source tube, $V_{b}$ was found to be equal to $50 \mathrm{~V}$ and $V_{\mathrm{ds}}$ was found to be equal to $34.5 \mathrm{~V}$. $V_{b}$ varies by less than $5 \%$ across the radius of the target plate and is therefore assumed constant at $50 \mathrm{~V}$. The ion beam velocity was therefore found to be $v_{b}$ $=15500 \mathrm{~m} \mathrm{~s}^{-1}$. The ions in the local plasma that enter the sheath on both the front and back sides of the MFMI target are accelerated and strike the target at the Bohm velocity which, since $T_{e}=5.2 \mathrm{eV}$ downstream, ${ }^{21}$ is $3530 \mathrm{~m} \mathrm{~s}^{-1}$.

From the RFEA measurement, the ratio of the ion beam density to the local downstream plasma density can also be determined and used to calculate the ion beam density. From Charles $^{30}$ the density ratio is

$$
\frac{n_{b}}{n_{\mathrm{ds}}^{+}}=\left(\frac{I_{b}}{I_{\mathrm{ds}}+I_{b}}\right) \frac{v_{\mathrm{Bohm}}}{v_{b-\mathrm{ds}}},
$$

where $I_{\mathrm{ds}}$ is the current collected by the REFA at the discriminator voltage corresponding to the local downstream plasma potential $V_{\mathrm{ds}}$ and $v_{b-\mathrm{ds}}=\sqrt{2 e\left(V_{b}-V_{\mathrm{ds}}\right) / m_{i}}$, the component of the ions velocity that comes from the difference between the potentials of the beam and the downstream plasma. For the conditions outlined above $V_{b}=50 \mathrm{~V}, V_{\text {local }}=34.5 \mathrm{~V}$, and $T_{e}$ is assumed constant at $5.2 \mathrm{eV}$ downstream of the HDLT source from previous measurements ${ }^{21}$ and therefore, $n_{b} / n_{\mathrm{ds}}^{+} \approx 0.1 . n_{b} / n_{\mathrm{ds}}^{+}$is also constant as a function of radius.

With the radial Langmuir probe positioned $1 \mathrm{~cm}$ in front of the MFMI target at $z=12 \mathrm{~cm}$, the probe is biased at $-73 \mathrm{~V}$ to be in the ion-collection mode. This allows the density to be determined using the ion-saturation-current expression,

$$
I_{\text {sat }}=0.6 e A_{\mathrm{LP}} v_{B} n,
$$

where $A_{\mathrm{LP}}$ is the area of the Langmuir probe, $n$ is the plasma density, and $v_{B}=\left(k T_{e} / m_{i}\right)^{1 / 2}$ where $k$ is Boltzmann's constant and $T_{e}$ is the electron temperature. The measured total density of the local downstream plasma is about $2.6 \times 10^{15} \mathrm{~m}^{-3}$. Since $n_{b} / n_{\mathrm{ds}} \approx 0.1$, the ion beam density at this position is $\sim 2.6 \times 10^{14} \mathrm{~m}^{-3}$ and $n_{\mathrm{ds}}^{+}$is about $2.3 \times 10^{15} \mathrm{~m}^{-3}$. The density measured behind the MFMI target, with the Langmuir probe $1 \mathrm{~cm}$ behind the target plate at $z=14 \mathrm{~cm}$, for these conditions is $2.0 \times 10^{15} \mathrm{~m}^{-3}$.

Using the information obtained from the diagnostic probes outlined above, the force on the target from the downstream plasma and the ion beam can be calculated. $F_{\mathrm{ds}}^{+}$and $F_{\mathrm{ds}}^{-}$, the forces on the front and back of the target from the downstream plasma, were found to be similar at 25.4 and $21.9 \mu \mathrm{N}$, respectively. In this case, the target was about 1 mean free path for ion-neutral charge exchange collisions from the exit of the HDLT source which ensured a very low ion beam density and force comparable to that from the neutrals. Using Eq. (12) and the measurements with the diagnostic probes, the calculated force from the ion beam was found to be $54.2 \mu \mathrm{N}$. Since $F_{p}=F_{b}+F_{\mathrm{ds}}^{+}-F_{\mathrm{ds}}^{-}$, the calculated force from the plasma is $F_{p}=57.7 \mu \mathrm{N}$.

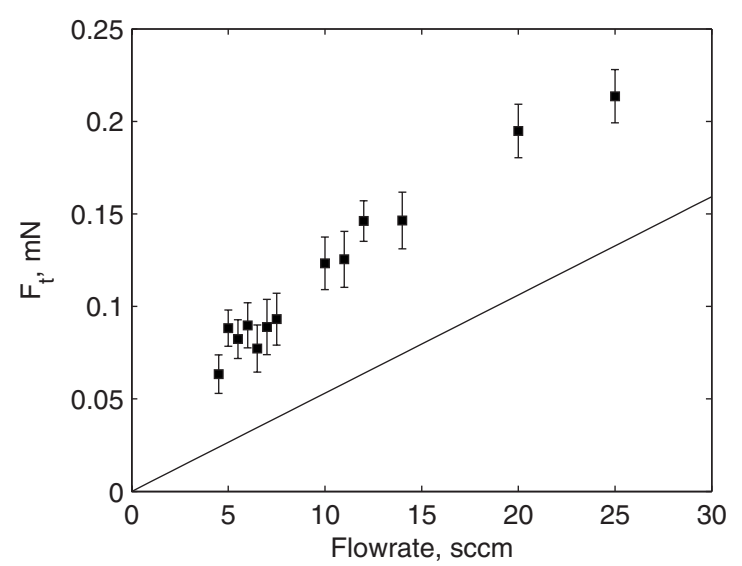

FIG. 8. Force on the target for a plasma formed at $100 \mathrm{~W}$ and $138 \mathrm{G}$ at varying flow rates (filled square). The calculated force from the neutrals $F_{n}$ is also shown (solid line).

For these operating conditions, the displacement of the reflected laser beam spot of the MFMI was measured and found to be $\Delta y=111 \pm 14$ units with the propellant flow alone and $\Delta y=214 \pm 17$ units with the HDLT operating and the plasma ignited. Using the calibration curve in Fig. 4, the force measured on the target with the propellant flow alone $F_{n}$ is $66.0 \pm 12.2 \mu \mathrm{N}$ and with the HDLT operating and the plasma ignited $F_{t}=123.3 \pm 14.2 \mu \mathrm{N}$. Therefore the force from the plasma $F_{p}$ measured by the MFMI is approximately $57.3 \mu \mathrm{N}$, which is in good agreement with the value of $F_{p}$ calculated from the information obtained from the measurements made with the diagnostic probes.

\section{Varying propellant flow rate}

To investigate the use of the MFMI under different operating conditions while remaining in a low thrust and hence high sensitivity range, measurements were made at various flow rates with the HDLT operating at $100 \mathrm{~W}$ of rf power and a maximum axial dc magnetic field inside the HDLT source of $138 \mathrm{G}$. The results are shown in Fig. 8 along with the calculated force from the neutrals $F_{n}$ plotted as a solid line.

The plasma parameters were also investigated under these conditions using the diagnostic probes positioned at $r=0 \mathrm{~cm}$ and while varying the propellant flow rate between 5 and $13.9 \mathrm{sccm}$. The RFEA was positioned in front of the MFMI target at $z=9 \mathrm{~cm}$ with the orifice facing the HDLT exhaust $\left(\theta=0^{\circ}\right)$ and the Langmuir probe was positioned $1 \mathrm{~cm}$ in front of the MFMI target $(z=12 \mathrm{~cm})$ and $1 \mathrm{~cm}$ behind the MFMI target $(z=14 \mathrm{~cm})$. From the RFEA measurements shown as $\square$ in Fig. 9, it is clear that the ion beam energy increases as the propellant flow rate is decreased and therefore, as per Eq. (13), the ion beam velocity also increases with decreasing flow rate. This result is consistent with previous studies with this plasma source and others. $^{21,31,32}$

Using the RFEA, the ratio of the ion beam density to the local downstream plasma density, $n_{b} / n_{\mathrm{ds}}^{+}$, calculated from Eq. (16), was determined and found to remain reasonably constant at 0.1 as the flow rate is varied. From the Langmuir probe measurements, the total density in front and behind the 


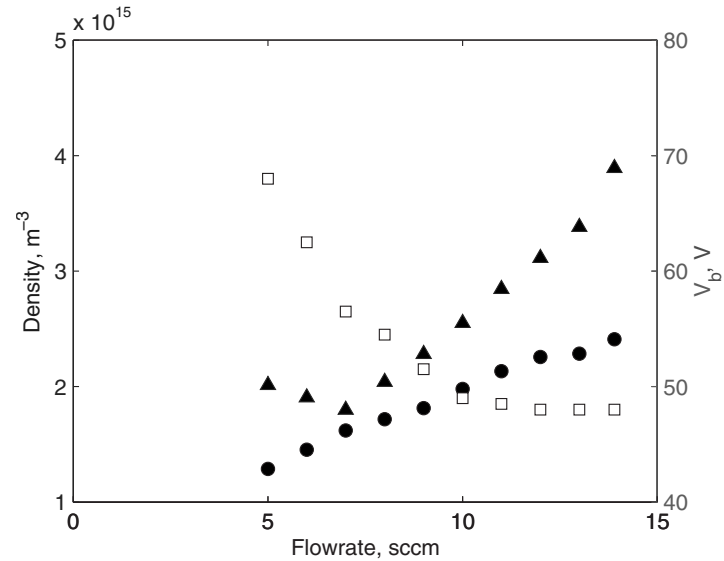

FIG. 9. The total plasma density measured with the Langmuir probe positioned in front of the MFMI target at $z=12 \mathrm{~cm}(\boldsymbol{\Delta})$ and behind the MFMI target at $z=14 \mathrm{~cm}(\boldsymbol{)})$ as a function of flow rate. The beam potential $\mathrm{V}_{b} \mathrm{vs}$ flow rate as measured with the RFEA at $z=9 \mathrm{~cm}(\square)$ is also shown.

MFMI target increases quasilinearly with increasing flow rate, as shown in Fig. 9. Hence $n_{b}$ and $F_{b}$ also increase with increasing flow rate [Eq. (12)].

Figure 10 shows the force from the plasma $F_{p}$ measured with the MFMI ( $\square$ ) and calculated from Eq. (14) using the measurements from the diagnostic probes $(\triangle)$ both as a function of flow rate. $v_{b}$ is calculated using $V_{b}$ measured with the RFEA and Eq. (13), and $T_{e}$ is assumed invariant with flow rate over this range, ${ }^{33}$ therefore $v_{\text {Bohm }}$ is constant at $3530 \mathrm{~m} \mathrm{~s}^{-1}$. The measurements obtained with the MFMI are in good agreement with those calculated using the information from the measured plasma parameters. These measurements demonstrate that variations in momentum flux and hence force from different ion beam velocities and plasma densities can be characterized with this instrument.

\section{CONCLUSION}

A high sensitivity MFMI that uses a laser displacement system has been developed for measuring low range thrust produced by electric propulsion devices that cannot easily be placed on conventional thrust balances. This system is a low

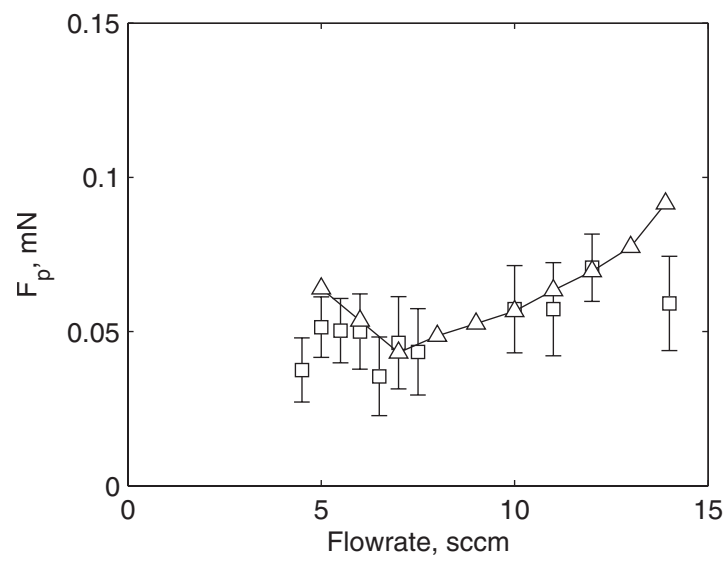

FIG. 10. The force from the plasma $F_{p}$ measured with the MFMI as a function of the flow rate $(\square)$. The force from the plasma, calculated using Eq. (14) and the information obtained from the diagnostic probes, as a function of flow rate $(\triangle)$. The solid line joining the data points is for clarity only. cost method that complements the use of conventional thrust balances. The instrument has been installed inside a vacuum chamber and calibrated via two different methods and is able to measure forces in the range of $0.02-0.5 \mathrm{mN}$ with a resolution of $15 \mu \mathrm{N}$. Measurements have been made of the force produced from the cold gas flow and with a discharge ignited using argon propellant. The plasma is generated using a HDLT prototype. The instrument target is placed about 1 mean free path for ion-neutral charge exchange collisions away from the open end of the HDLT prototype. At that position the plasma consists of a low density ion beam and a much larger downstream component. The results are in good agreement with those determined from the plasma parameters measured with diagnostic probes and measurements at various flow rates show that variations in ion beam velocity and the resulting momentum flux can be measured with this instrument. The measurement approach used here is free from $r f$ interference and thermal effects as the laser displacement system is placed outside the vacuum chamber. This instrument could also be used to measure the thrust in the exhaust of other electric propulsion devices ${ }^{34-36}$ and the momentum flux of ion beams formed by expanding plasmas 37,38 or fusion experiments. ${ }^{4}$

${ }^{1}$ S. A. Cohen, F. Zonca, J. Timberlake, T. Bennett, J. Cuthbertson, W. Langer, and R. Motley, Rev. Sci. Instrum. 61, 3586 (1990).

${ }^{2}$ I. S. Nedzelskiy, C. Silva, H. Fernandes, P. Duarte, and C. A. F. Varandas, Rev. Sci. Instrum. 78, 123505 (2007).

${ }^{3}$ J. Grun and B. H. Ripin, Rev. Sci. Instrum. 53, 1878 (1982).

${ }^{4}$ S. F. Goncharov, P. P. Pashinin, V. Y. Perov, R. V. Serov, and V. P. Yanovsky, Rev. Sci. Instrum. 59, 709 (1988).

${ }^{5}$ T. F. Yang, P. Liu, F. R. Chang-Diaz, H. Lander, R. A. Childs, H. D. Becker, and S. A. Fairfax, Rev. Sci. Instrum. 66, 4637 (1995).

${ }^{6}$ D. G. Chavers, F. R. Chang-Diaz, C. Irvine, and J. P. Squire, J. Propul. Power 22, 637 (2006).

${ }^{7}$ B. W. Longmier, B. M. Reid, A. D. Gallimore, F. R. Chang-Diaz, J. P. Squire, T. W. Glover, G. Chavers, and E. A. Bering, J. Propul. Power 25, 746 (2009).

${ }^{8}$ D. G. Chavers and F. R. Chang-Diaz, Rev. Sci. Instrum. 73, 3500 (2002).

${ }^{9}$ T. Lunt, C. Silva, H. Fernandes, C. Hidalgo, M. A. Pedrosa, P. Duarte, H. Figueiredo, and T. Pereira, Plasma Phys. Controlled Fusion 49, 1783 (2007).

${ }^{10}$ C. Charles, R. Boswell, and H. Kuwahara, Appl. Phys. Lett. 67, 40 (1995).

${ }^{11}$ C. Charles and R. Boswell, J. Appl. Phys. 84, 350 (1998).

${ }^{12}$ M. Bicker, U. von Hulsen, U. Laudahn, A. Pundt, and U. Geyer, Rev. Sci. Instrum. 69, 460 (1998).

${ }^{13}$ C. Fitz, W. Fukarek, A. Kolitsch, and W. Moller, Surf. Coat. Technol. 128, 474 (2000).

${ }^{14}$ V. Au, C. Charles, and R. W. Boswell, Appl. Phys. Lett. 88, 234103 (2006).

${ }^{15}$ E. A. Cubbin, J. K. Ziemer, E. Y. Choueiri, and R. G. Jahn, Rev. Sci. Instrum. 68, 2339 (1997).

${ }^{16}$ L. D. Cassady, A. D. Kodys, and E. Y. Choueiri, Proceedings of the 38th AIAA/ASME/SAE/ASEE Joint Propulsion Conference, 2002 (unpublished), p. 4118.

${ }^{17}$ A. D. Kodys, R. Murray, L. D. Cassady, and E. Y. Choueiri, Proceedings of the 42nd AIAA/ASME/SAE/ASEE Joint Propulsion Conference, 2006 (unpublished), p. 4821.

${ }^{18}$ C. R. Phipps, J. R. Luke, W. Helgeson, and R. Johnson, AIP Conf. Proc. 830, 492 (2006)

${ }^{19}$ M. A. Kemp and S. D. Kovaleski, IEEE Trans. Plasma Sci. 36, 356 (2008).

${ }^{20} \mathrm{~J}$. Li and Z. Tang, Chin. Opt. Lett. 3, 76 (2005).

${ }^{21}$ M. D. West, C. Charles, and R. W. Boswell, J. Propul. Power 24, 134 (2008).

${ }^{22}$ C. Charles, R. W. Boswell, and M. A. Lieberman, Appl. Phys. Lett. 89, 261503 (2006)

${ }^{23}$ G. D. Conway, A. J. Perry, and R. W. Boswell, Plasma Sources Sci. 
Technol. 7, 337 (1998).

${ }^{24}$ C. Charles, A. W. Degeling, T. E. Sheridan, J. H. Harris, M. A. Lieberman, and R. W. Boswell, Phys. Plasmas 7, 5235 (2000).

${ }^{25}$ C. Charles, D. Ramdutt, P. Brault, A. Caillard, D. Bulla, R. Boswell, H. Rabat, and A. Dicks, Plasma Phys. Controlled Fusion 49, A73 (2007).

${ }^{26}$ C. Charles, R. Boswell, A. Bouchoule, C. Laure, and P. Ranson, J. Vac. Sci. Technol. A 9, 661 (1991).

${ }^{27}$ C. Charles, R. W. Boswell, and R. K. Porteous, J. Vac. Sci. Technol. A 10, 398 (1992)

${ }^{28}$ W. Cox, C. Charles, R. W. Boswell, and R. Hawkins, Appl. Phys. Lett. 93, 071505 (2008).

${ }^{29}$ C. Charles and R. W. Boswell, Phys. Plasmas 11, 1706 (2004).

${ }^{30}$ C. Charles, Appl. Phys. Lett. 84, 332 (2004).

${ }^{31}$ M. A. Lieberman and C. Charles, Phys. Rev. Lett. 97, 045003 (2006).
${ }^{32}$ M. A. Lieberman, C. Charles, and R. W. Boswell, J. Phys. D: Appl. Phys. 39, 3294 (2006).

${ }^{33}$ C. Charles, Phys. Plasmas 12, 044508 (2005).

${ }^{34}$ C. Charles, R. Boswell, P. Alexander, C. Costa, O. Sutherland, L. Pfitzner, R. Franzen, J. Kingwell, A. Parfitt, P.-E. Frigot, J. Del Amo, and G. Saccoccia, IEEE Trans. Plasma Sci. 36, 1196 (2008).

${ }^{35}$ M. Inutake, A. Ando, K. Hattori, H. Tobari, T. Makita, M. Shibata, Y. Kasashima, and T. Komagome, Plasma Phys. Controlled Fusion 49, A121 (2007).

${ }^{36}$ L. Blackhall and J. Khachan, J. Phys. D: Appl. Phys. 40, 2491 (2007).

${ }^{37}$ C. S. Corr, J. Zanger, R. W. Boswell, and C. Charles, Appl. Phys. Lett. 91, 241501 (2007).

${ }^{38}$ C. S. Corr, R. W. Boswell, C. Charles, and J. Zanger, Appl. Phys. Lett. 92 , 221508 (2008). 